\title{
Experimental validation of the temperature behavior of the ENDF/B-VIII.0 thermal scattering kernel for light water
}

\author{
Jose Ignacio Marquez Damian ${ }^{1, *}$, Javier Dawidowski ${ }^{1}$, Jose Rolando Granada ${ }^{1}$, Florencia Cantargi ${ }^{1}$, Giovanni \\ Romanelli $^{2}$, Christian Helman ${ }^{1}$, Matthew Krzystyniak ${ }^{2}$, Goran Skoro $^{2}$, and Danila Roubtsov ${ }^{3}$ \\ ${ }^{1}$ Neutron Physics Department, Centro Atomico Bariloche - Comision Nacional de Energia Atomica, Argentina \\ ${ }^{2}$ ISIS Facility, Rutherford Appleton Laboratory, Chilton, Didcot, Oxfordshire OX11 0QX, United Kingdom \\ ${ }^{3}$ Canadian Nuclear Laboratories, Chalk River, ON K0J 1J0, Canada
}

\begin{abstract}
The Neutron Physics Department at Centro Atómico Bariloche developed new models for the interaction of thermal neutrons with water which have been validated against experimental data, including new thermal scattering experiments, and were adopted for the release of ENDF/B-VIII.0. Although the older models are, in general, good for most applications, some discrepancies had appeared in the case of heavy water, and this motivated new measurements that validated the new model. In the case of light water, the new model predicts a reduction of the total cross section around $0.025 \mathrm{eV}$ when the temperature is increased from room temperature. This reduction, that is not predicted by the existing models, and potentially affects the calculation of temperature reactivity coefficients in nuclear reactors, has been traced to a shift in the vibrational frequency spectrum of liquid water. The only experimental data previously available is from an experiment performed at the Demokritos reactor in the ' 60 s at $293 \mathrm{~K}$ and $473 \mathrm{~K}$, which validates the new model when the cross section ratios are computed. In order to verify this effect at a lower temperature range, a transmission experiment was carried out at the VESUVIO spectrometer in the ISIS facility in the UK in June 2018, measuring the total neutron cross section in the range from $283 \mathrm{~K}$ to $353 \mathrm{~K}$. Here, we present this new experimental data and its comparison with the models.
\end{abstract}

\section{Introduction}

The nuclear data library ENDF/B-VIII.0 [1] incorporates new evaluations for the thermal neutron scattering libraries for light and heavy water. These libraries were derived from models developed at Centro Atómico Bariloche, known as the CAB Models for Water [2], which are based on molecular dynamics results as well as on experimental data. The applications of these libraries to nuclear criticality benchmarks at room temperature help to reduce the bias previously found in the calculation of water moderated reactor benchmarks $[1,3]$.

The CAB Models introduce differences in the calculations of the total cross section and derived quantities, which need to be verified experimentally. In the case of heavy water, there was a discrepancy in the calculated values of the total cross section, and new transmission [4] and scattering [5] experiments helped to verify the validity of the new model.

One of the characteristics of these models is the temperature dependence of the rotational spectra, which in the case of light water leads to a reduction of the total cross section around $0.025 \mathrm{eV}$ when the temperature is raised from room temperature. A similar effect was observed experimentally by Dritsa and Kostikas [6] in total cross sec-

*e-mail: marquezj@cab.cnea.gov.ar tion measurements performed at the Demokritos reactor (Greece) at $T=293 \mathrm{~K}$ and $473 \mathrm{~K}$.

In order to verify this effect, a transmission experiment was carried out [7] at the VESUVIO spectrometer [8] in the ISIS facility in the UK in June 2018. The experiment was designed to measure the variation of the thermal neutron total cross section of light water $\left(\mathrm{H}_{2} \mathrm{O}\right)$ in the unpressurized range, at temperatures between $283 \mathrm{~K}$ and $353 \mathrm{~K}$. The expected difference in the total cross section between these two temperature points was of $\sim 1 \%$, which requires a high count rate to reach statistical significance. Previous experiments on the same instrument [9] had shown this requirement to be achievable.

\section{Experimental method}

The VESUVIO spectrometer at target station 1 (TS1) of the ISIS spallation neutron source at the Rutherford Appleton Laboratory (UK) was used in a configuration for neutron transmission experiments. The samples were nanopure water contained inside of a standard flat-side aluminum sample holder, with a 1-mm-thick spacer and teflon internal coatings to reduce oxidation (Fig. 1). The sample holder was filled with water, sealed and tested in a vacuum oven for 24 hours at $353 \mathrm{~K} / 50$ mbar to ensure that no sample was lost. Additional details of the instrument configuration can be found in Ref. [9]. 
Heating elements and a thermocouple were attached to the sample holder outside of the beam path, and the sample was placed inside a closed circuit refrigerator (CCR) to control temperature. The sample was stabilized within $0.1 \mathrm{~K}$ before opening the beam.

Two main measurements at $283 \mathrm{~K}$ and $353 \mathrm{~K}$ were performed, using accelerator beam time of $3515 \mu \mathrm{Ah}(\approx$ 20 hours) per measurement. A shorter measurement of $1080 \mu \mathrm{Ah}(\approx 6$ hours) was performed at $293 \mathrm{~K}$ to compare with previous measurements. After the sample measurements were done, the cell was emptied and measured for $2350 \mu \mathrm{Ah}$ ( $\approx 13$ hours). Background count was measured using a cadmium plate of $1.5 \mathrm{~mm}$ thick.

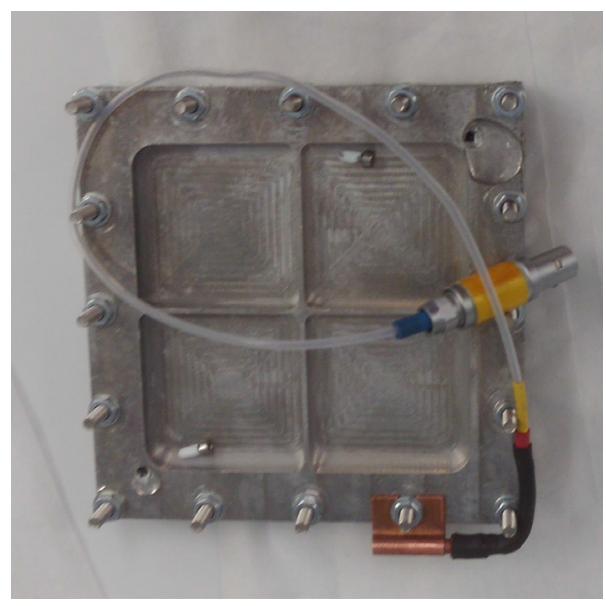

Figure 1. Photograph of the sample holder while it was loaded with water.

\section{Data processing and results}

\subsection{Data processing}

Time-of-flight spectra for the samples at $283 \mathrm{~K}, 293 \mathrm{~K}$, and $353 \mathrm{~K}$, empty cell and background were processed using the data reduction and analysis application Mantid $[10,11]$.

The measured transmission spectra $S(E)$ (sample), $F(E)$ (empty cell) and $B(E)$ (background), were summed and normalized to an integral of the incident beam monitor. The logarithm of the transmission $\tilde{\sigma}(E)$ was computed as follows

$$
\tilde{\sigma}(E)=-\ln \frac{S(E)-B(E)}{F(E)-B(E)} .
$$

The resulting values were fitted in the epithermal energy range $(0.5 \mathrm{eV}<E<10 \mathrm{eV})$ with the function $f(E)=A_{1}\left(1+A_{2} / E\right)$, which is the asymptotic form of the total scattering cross section [12]. The fitting parameters were used to normalize $\tilde{\sigma}(E)$ to the asymptotic value of the total cross section, $\sigma_{\text {free }}^{\mathrm{H}_{2} \mathrm{O}}=44.67 \mathrm{~b}$, to match the free gas values of $\mathrm{H}$ and $\mathrm{O}$ in the ENDF/B-VIII.0 library,

$$
\sigma(E)=\frac{\sigma_{\text {free }}^{\mathrm{H}_{2} \mathrm{O}}}{A_{1}} \tilde{\sigma}(E) .
$$

The results are shown in Fig. 2.

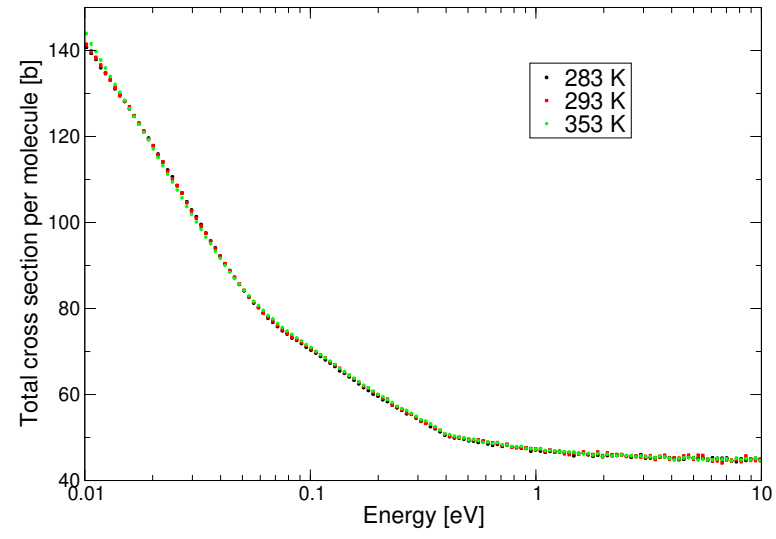

Figure 2. Experimental total cross section for light water at the three measured temperature points.

Fig. 3 shows the measurements at room temperature, compared with reference data from Russell [13] (EXFOR entry 11162003). The two sets are found to be equivalent within the experimental uncertainty.

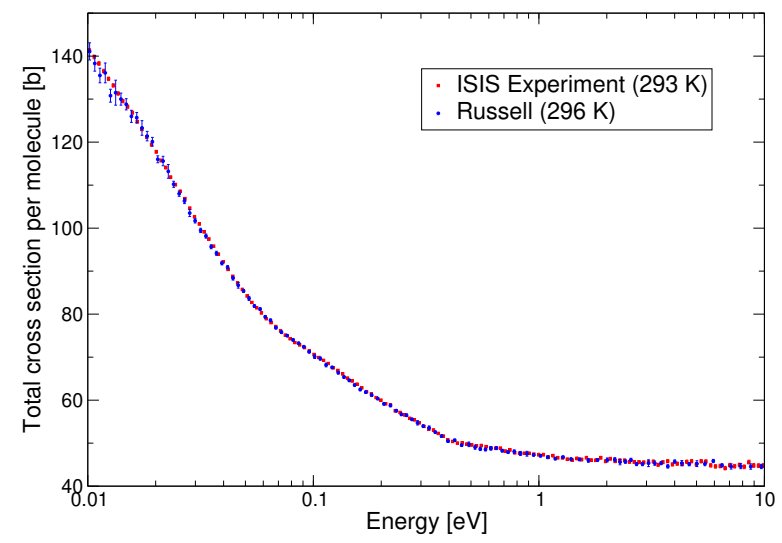

Figure 3. Comparison of experimental total cross section for light water at room temperature.

\subsection{Comparison with models}

A comparison of the measured and calculated difference between the values of two total cross sections, $\sigma\left(E ; T_{2}\right)$ and $\sigma\left(E, T_{1}\right)$, is given in Fig. 4 when the temperature is raised from $T_{1}=283 \mathrm{~K}$ to $T_{2}=353 \mathrm{~K}$. For reference, the calculated difference between the older ENDF/B-VII.1 evaluation [14] at $350 \mathrm{~K}$ and $293 \mathrm{~K}$ is shown in the same plot.

In the energy range $0.015 \mathrm{eV}<E<0.050 \mathrm{eV}$, a reduction of the total cross section is observed. This difference has a minimum of $-1.1 \pm 0.1 \mathrm{~b}$ in the range $0.025 \mathrm{eV}<E<0.030 \mathrm{eV}$. This range of decrease is followed by an increase of the cross section in the range of $0.050 \mathrm{eV}<E<1 \mathrm{eV}$. In the epithermal range the difference tends asymptotically to zero, in agreement with the expected value in the free gas limit. Below $0.015 \mathrm{eV}$, the 
measurement shows an increase of the total cross section when the temperature is raised, which is compatible with the greater mobility of molecules caused by a reduction of the diffusion coefficient.

The new model correctly predicts the decrease in the cross section of $1.1 \mathrm{~b}$ in the energy range $0.025 \mathrm{eV}<$ $E<0.030 \mathrm{eV}$ when the temperature rises from $283 \mathrm{~K}$ to $353 \mathrm{~K}$, an effect that was not observed in calculations performed with older models of the thermal neutron scattering on light water and with evaluations different from the ENDF/B-VIII.0. The new model also reproduces the oscillations observed in the $0.1-0.3 \mathrm{eV}$ energy range, although a small discrepancy between calculated and experimental values less than $0.2 \mathrm{~b}$ suggests that the high energy region of the rotational band of $\mathrm{H}_{2} \mathrm{O}$ could be further improved in the model.

\section{Conclusions}

An experiment was carried out to measure the total cross section of light water in the $0.01-10 \mathrm{eV}$ energy range, at 283, 293 and $353 \mathrm{~K}$. The results at room temperature are compatible with previous experiments, and the temperature variation shows changes in the total cross sections clearly observable with the achieved statistics. In particular, at the energy range $0.025 \mathrm{eV}<E<0.030 \mathrm{eV}$, a decrease of $1.1 \pm 0.1 \mathrm{~b}$ when the temperature rises to 353 $\mathrm{K}$ is observed, which confirms the experimental observations by Dritsa and Kostikas (1967). At present the CAB Model of water (incorporated in the ENDF/B-VIII.0 evaluated nuclear data library), is the only model that explains this unusual feature in the behaviour of the total cross sections. This result contributes to the validation of the new light water thermal neutron scattering evaluation included in that library.

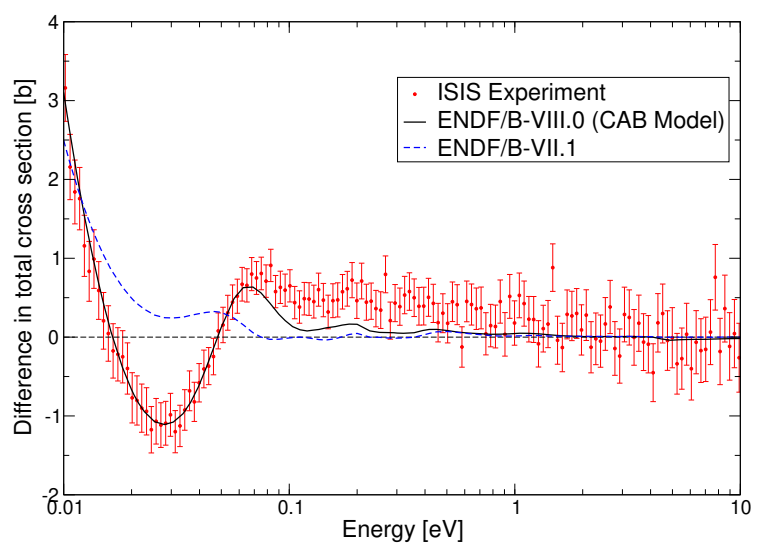

Figure 4. Comparison of the measured and calculated difference in the total cross section for light water at $353 \mathrm{~K}$ and $283 \mathrm{~K}$, this is, $\sigma(E ; 353 \mathrm{~K})-\sigma(E ; 283 \mathrm{~K})$ vs. $E$.

\section{Acknowledgments}

The authors are grateful to the UK Science and Technologies Facilities Council for the beam time allocated to this experimental proposal (RB1810191).

\section{References}

[1] D.A. Brown, M. Chadwick, R. Capote, A. Kahler, A. Trkov, M. Herman, A. Sonzogni, Y. Danon, A. Carlson, M. Dunn et al., Nuclear Data Sheets 148, 1 (2018)

[2] J.M. Damian, J. Granada, D. Malaspina, Annals of Nuclear Energy 65, 280 (2014)

[3] J.M. Damian, J. Granada, D. Roubtsov, Annals of Nuclear Energy 71, 206 (2014)

[4] J. Márquez Damián, J. Granada, D. Baxter, S. Parnell, D. Evans, Nuovo Cimento C 38 (2016)

[5] G. Li, G. Bentoumi, K. Hartling, I. Molnar, C. Neggers, R. Rogge, Z. Yamani, Annals of Nuclear Energy 135, 106932 (2020)

[6] M. Dritsa, A. Kostikas, EANDC (OR)-63L (1967)

[7] J. Dawidowski, et al, On the behavior of light water cross section with temperature, https://doi .org/ 10. 5286/ISIS. E. RB1810191

[8] G. Romanelli, M. Krzystyniak, R. Senesi, D. Raspino, J. Boxall, D. Pooley, S. Moorby, E. Schooneveld, N. Rhodes, C. Andreani et al., Measurement Science and Technology 28, 095501 (2017)

[9] L.R. Palomino, J. Dawidowski, J.M. Damián, G. Cuello, G. Romanelli, M. Krzystyniak, Nuclear Instruments and Methods in Physics Research Section A: Accelerators, Spectrometers, Detectors and Associated Equipment 870, 84 (2017)

[10] M. Mantid, Mantid Project (2013)

[11] O. Arnold, J.C. Bilheux, J. Borreguero, A. Buts, S.I. Campbell, L. Chapon, M. Doucet, N. Draper, R.F. Leal, M. Gigg et al., Nuclear Instruments and Methods in Physics Research Section A: Accelerators, Spectrometers, Detectors and Associated Equipment 764, 156 (2014)

[12] G. Placzek, Physical Review 86, 377 (1952)

[13] J. Neill, J. Russell Jr, J. Brown, Nuclear Science and Engineering 33, 265 (1968)

[14] M. Chadwick, M. Herman, P. Obložinskỳ, M.E. Dunn, Y. Danon, A. Kahler, D.L. Smith, B. Pritychenko, G. Arbanas, R. Arcilla et al., Nuclear data sheets 112, 2887 (2011) 